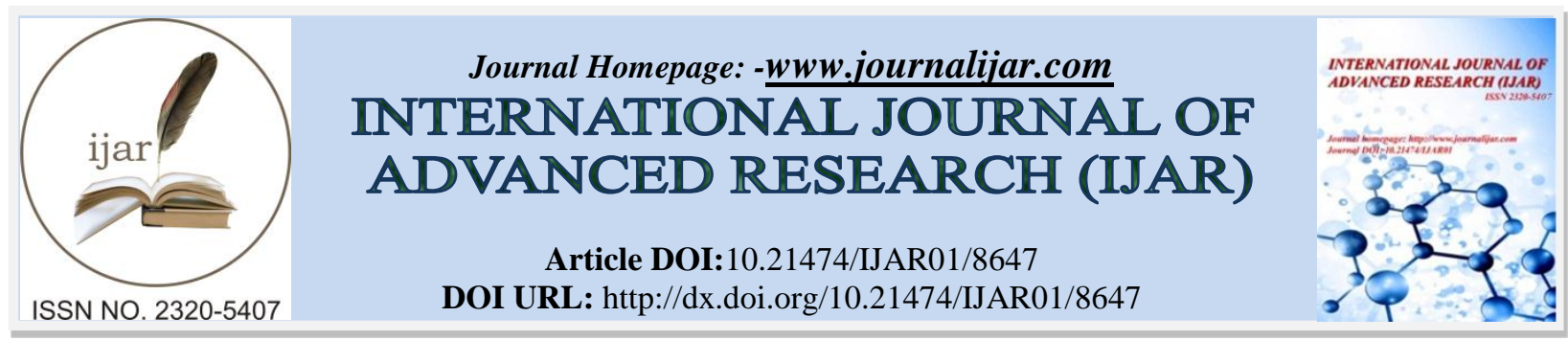

RESEARCH ARTICLE

\title{
RELATIONSHIP BETWEEN THE JNK PATHWAY AND APOPTOSIS IN HEART FAILURE.
}

\section{Zhao Junpeng $^{1}$, Tian Guozhong ${ }^{2}$, Ahmad Zaki Barati ${ }^{3}$ and Santosh Adhikari ${ }^{4}$.}

1. Department of human antomy and histoembryology, Jiamusi University, Jiamusi,China.

2. College of basic Medicine, Jiamusi University, Jiamusi, China.

3. Department of Orthopedics, First affiliated Hospital of Jiamusi University, Jiamusi, China.

4. Department of Pediatrics, First affiliated Hospital of Jiamusi University, Jiamusi, China.

\section{Manuscript Info}

Manuscript History

Received: 08 January 2019

Final Accepted: 10 February 2019

Published: March 2019

Key words:-

JNK, Heart failure, Apoptosis.

\begin{abstract}
JNKs belong to mitogen activated protein kinases family and are involved in regulation of various cellular responses including cell survival, cell proliferation and differentiation. They can be activated by several factors such as radiation, stress, growth factors, cytokines and extracellular signals. JNKs activation results in phosphorylation of downstream molecules involve in normal cellular processes.

Furthermore the recent data have linked JNK signaling with different pathological coditions, including inflammation, cancer , neurodegenrative disease and cardiac hypertrophy. Gq signaling initiates hypertrophic growth of neonatal rat cultured myocytes and is postulated to transduce in vivo cardiac pressure overload hypertrophy.however with the over expression of c-jun through Ap-1 and ATF-2 transcripting factor, it can cause decompensatory hypertrophy which can proceed to cardiac failure. This review analyzes our present understanding of the role of JNK in apoptotic signaling in heart failure and some other mechanism which induces apoptosis in cardiomyocytes.
\end{abstract}

Copy Right, IJAR, 2019,. All rights reserved.

\section{Introduction:-}

JNK was discovered as a second subfamily of MAPKs for its ability to phoshorylate microtubule associated protein in rate liver following cycloheximide injection.MAPK(Mitogen activated protein kinase) pathways play a significant role in mediating apoptosis and cell growth[1] Some studies suggested that members of the MAPK family, including p38-MAPKs, c-Jun NH2-terminal kinase (JNK) and extracellular signal-regulated kinase (ERK) play key roles in caridomyocyte apoptosis and regulating the hypertrophy in regulating hypertrophy[2].

JNKs are stress activated protein kinase that is related to the MAPK(Mitogen activated protein kinase), that also includes the p38, ERKs MAPKs [3,4]. Whether the activation of JNKs causes the cell proliferation or apoptosis is dependent on variety of stimuli and cell type involve in such activation $[5,6]$. JNKs were identified by their ability to phosphorylate c-Jun in response to stress induced by a physical stimulation and the different chemicals such as heat sock, osmotic stress, Uv radiation and TNF[7]. Some studies suggested that JNKs can be activated by variety of stimuli such as the growth factor,cytokines and stress factors[8]. 
JNKs are activated by MAP2 kinases such as MKK4/7 [9], regulate the apoptosis[10], respond to stresses[11]and proliferation[12]There are two groups of signaling pathways that initiates the apoptosis 1) Extrinsic pathway that starts signaling by death receptors such as TNF-a TRAIL FAS -1and 2. 2) Intrinsic pathway that stimulated by mitochondrial events [11]. JNK plays focal role in both following pathways. There are three different types of JNK, namely JNK1, JNK2, and JNK3[12,13] .JNKs form the last tier of the three-tier kinase module consisting of MAP kinase kinase kinase (MAP3K), MAP kinase kinase (MAP2K), and MAP kinase (MAPK)[14,15].In response to the different stimuli, the penult dual specificity kinase of tier, either MKK7 or MKK4, actives JNK by phosphorylation Thr- and Thr residue of TXY motif, within the activation loop of the JNKs[15]. While MKK4 can activate both p38MAPK and JNK, MKK7 is specifically involved in the activation of JNKs.Both anti-apoptotic and pro-apoptotic signals converge on activating MKK4-JNK or MKK7-JNK signaling node via specific MAP3Ks. JNKs in turn activate apoptotic signaling either through the upregulation pro-apoptotic genes via the transactivation of specific transcription factors including c-Jun or by directly modulating the activities of mitochondrial pro- and anti-apoptotic proteins through phosphorylation events[16].The MKK7 activates the specific JNK, bothe the proapoptotic signals.Some studies suggest that oxidative stress is a major apoptotic stimulus in different heart diseases MacLellan WR, Schneider MD. Death by design. Circ Res 1997;81:137-44.About 25 years ago, Jun kinase was introduced as the member of the PP54 microtubule associated protein-2 kinases. This review analyzes our current understanding of the mechanisms through which JNK-signaling causes cell dead or apoptosis in heart failure.

\section{Overview of Jnk}

JNK is one of the mitogen activated protein kinase pathways that transudce extracellular signals to control the processes such as the differentiation, migration, cell proliferation and apoptosis[17].Each of MAPK pathways are activated through the phosphorylation reations. When there is upstream of jnk MAP3K family members phosphoryate and activate the MAP2K engzymes(MKK4 and MKK7), that in turn, it phosphorylate and activate the JNK.The pathway which is facilitated by the scaffold proteins such as the JNK interacting protein 1(JIP1) will rapidly activates[18]. Whereas the JNK inactivation pathways occurs by dephosphorylation performed by a group of dual specificity phosphatases[19]. The JNK can phosphorylate threonin and serine residues in a specific protein subrates upon its activation. JNK obtained its name from its ability to phosphorylate serine residues at positions 63 and 73 in the amino-terminal domain of the c-Jun proto-oncogene [20].JNK family has three genes (Jnk1/Mapk8, Jnk2/Mapk9, and Jnk3/Mapk10). JNK1 and JNK2 enzymes are expressed in most cells of the body, including the kidney, whereas expression of JNK3 is in the heart, brain, and testis[21]. Deletion of both Jnk1 and Jnk2 genes causes fetal lethality, whereas deletion of either Jnk1 or Jnk2 results in viable and healthy mic[22].Alternative joinning of mRNA from these three genes gives rise to at least 10 different JNK enzyme isoforms ranging between 46 and $55 \mathrm{kDa}$ [23].It is suggested that the JNK pathway can be activated by the specific stimulation that can activate distict cellular pathways and responses[24]. An important aspect of the is that it can activate through stimuli such as inflammatory cytokines, stress, Oxidative oxygen species. Thus the JNK signaling may contribute to heart failure through the apoptosis.

\section{JNK signaling in myocardium}

Within the heart JNK pathways can be activate in response to distinct stimuli by stressors such as the oxidative stress, DNA damage,Hypoxia,ischemia reperfusion(IR), hyperosmolality, ER stress, Inflammtory cytokines and UV radiation[25].G protein coupled receptors, groth factors, non canonical Wnt signaling can also induce the JNK signaling pathways[26], the latter being critical in cardiac develpment[27].During the heart develpment JNK pathways can be activated throught the non canonical Wnt signaling which also implicated in determination of cardiac cell fate and morphogenesis of heart, including proper development of outflow tract [28].During cardiac disease JNK can be activated through the I/R injury[29]. During I/R injury and intracellular pathways when the cells within in myocardium undergo apoptosis, through which ischemic induces cell death and other "stress responses" in cardiomyocytes have been extensively charaterized. JNK signaling cascade can be initiated by the MAPK pathway, that activated during the reperfusion that results in to activation of the AP-1 trascription factor and subsequently causes cellular apoptosis[30].On the other hand it can also activate in heart in response to different pathological conditions such as hypertension, myocardial infarction, reperfusion injury and inschemia[31].Different studies have show that JNK signaling is shown to be connected with key mediator pathoogical caridac remodeling and heart failure. It has been shown that JNK plays a vital role in cardiac hypertrophy and cardiomyocyte apoptosis[32].

The three most distict forms of JNKs, are involved in stimulating apoptotic signaling cascade.Furthure more the clue that shows that JNKs are involved in apoptotic singaling process came form the of $\gamma$-ray stimulated the activation of JNK-1 along with an increase in DNA fragmentation in Jurkat cells[33]. While $\gamma$-ray induced JNK-activation was 
delayed, the growth factor induced JNK-activation was rapid and transient. Subsequent studies expanded the role of JNK in TNF- $\alpha$, Fas-L, X-ray, and UV-ray induced apoptosis in different cellular and physiological contexts. Unequivocal evidence that JNK1 and JNK2 are involved in apoptotic signaling came from studies using embryonic fibroblasts derived from JNK1-/-JNK2-/- mice that showed resistance to apoptosis in response to diverse genotoxic and cytotoxic stress [34,35]. In general the mechanism through which JNKs regulates the apoptotic pathway involves expression of pro-apoptotic genes by activating the specific transcrition factors such as the c-Jun, p73 and p53 [36].

\section{Nuclear signaling of JNK in the regulation of apoptosis}

Upon activation by the upstream MAP2Ks, the phosphorylated JNK translocates to the nucleus where it phosphorylates and transactivates c-Jun [36] resulting in the formation of AP-1. The AP-1 is involved in the transcription of a wide variety of proteins, including several pro-apoptotic proteins [37] such as TNF- $\alpha$, Fas-L, and Bak [38]. JNK can also phosphorylate several other transcription factors including JunD, ATF2, ATF3, Elk 1, Elk-3, p53, RXR $\alpha$, RAR $\alpha$, AR, NFAT4, HSF1, and c-Myc [39]. Thus, in the context of apoptosis, the nuclear activity of JNK can potentially lead to an increase in the expression of pro-apoptotic genes and/or a decrease in the expression of pro-survival genes. There is sufficient evidence that the nuclear activity of JNK and the transactivation of c-Jun are required for its apoptotic activity. It has been observed that JNK is required for the apoptosis of central nervous system neurons and the expression of dominant negative inhibitors of nuclear JNK confer resistance to their apoptosis following trophic support [40]. These findings suggest an important role of nuclear JNK in promoting apoptotic signaling. It is significant to note here that the non-phosphorylatable mutants of c-Jun confer resistance to apoptosis of MEFs in response to UV-irradiation. Together, these results may be indicative of a role for JNK-cJun/Ap1 mediated expression of pro-apoptotic genes in JNK-mediated apoptosis. An alternate pathway contributing to JNKmediated apoptosis involves the phosphorylation of the p53 family of proteins by JNK [41]. It has been suggested that the phosphorylation of p53 at Ser6 by JNK inhibits ubiquitin-mediated degradation of p53 and thereby stabilizing the levels of p53. Recent studies have shown that the phosphorylation of p53 at Ser6 by JNK2 is critically required for the apoptotic pathway [42].

It has been observed that the expression of FDH (10-formyltetrahydrofolate dehydrogenase) is drastically reduced in tumors and its elevation induces p53-dependent apoptosis. Further analyses of this apoptotic event indicated that FDH induces direct phosphorylation of p53 at Ser6 involving both JNK1 and JNK2. Treatment of FDH-expressing cells with JNK inhibitor SP600125 or silencing of JNK1/2 or inhibited the phosphorylation of p53 at Ser6 as well as p53-dependent apoptosis in response to DNA-damage. Since p53 is involved in the expression of several proapoptotic genes such as Bax (Bcl2-associated X protein) and PUMA (p53 up-regulated modulator of apoptosis), it is quite possible that apoptotic pathway activated by JNK involves p53-mediated upregulation of pro-apoptotic genes. DNA damage that activates JNK also causes the stabilization and activation of p73, a member of the p53 family of transcription factors [43]. Similar to p53, p73 induces apoptosis by increasing the expression of proapoptotic genes including Bax and PUMA. Analysis of this pathway has shown that JNK is required for p73-mediated apoptosis induced by the DNA damaging agent cisplatin and it involves the phosphorylation of p73 at several serine and threonine residues. Consistent with the notion that JNK and its activity are required for p73-mediated apoptosis, mutation of JNK-phosphorylation site in p53 abrogates cisplatin-induced stabilization of p73 along with a marked reduction in p73- transcriptional activity, and cisplatin-induced apoptosis. Thus, JNK can stimulate the expression of pro-apoptotic genes and decrease the expression pro-survival genes via multiple transcription factors in cell-type and stimuli specific manner.

\section{JNK in Intrinsic Apoptotic Pathway}

Cytoplasmic stress and the damage to the DNA activates pro- apoptotic protein Bak or Bax throgh the Bh3 protein, which in turn it induces the mitochondrial outer membrane permeabilization, resulting in release of the pro-apoptotic proteins such as smac/diablo and omi/htra2 and the Cytochrome $\mathrm{C}$ from mitochondrial intermembrane space.

Release cytocrome c, together with Apaf-1 and caspase 9 from the apoptosomes, that triggers caspase- 9 leading to the activation of caspase 3 and -7 which results in to cellular apoptosis.Further more the following process potentiated by the OMI and SMAC that inhibits the anti apoptotic protein XIAP. The observation in which the MOMB and the consequesnt release of cytochrome $\mathrm{c}$ in reponse to UV irradiation were inhibited in JNK1-/- and JNK2/- MEFsSuggests a role for the kinases in the intrinsic pathway[44].One mechanism through which JNK mediates the release of mitochondrial cytochrome $\mathrm{C}$ is through its ability to increase the expression of $\mathrm{BAX}$ via transcriptional activation of c-Jun leading to MOMB [45]. JNK triggers the release of cytochrome c through stimulating traslocation of Bax to mitochondria[46]. JNK mediates translocation of Bax by phosphorylation of the 
phosphorylation of 14-3-3 $\alpha$ and/ or 14-3-3 $\zeta$ protein. Phosphorylation of the 14-3-3 proteins leads to dissociation of BAX following which it translocates to mitochondria to induce MOMB and cytochrome c release. Moreover, to promote its translocation to mitochondria and subsequent release of cytochrome c JNK directly phosphorylates 143-3 associated Bad. To promote intrinsic apoptosis further JNK targeting BH3-only family of pro-apoptotic proteins [47]. JNK phosphorylates the pro-apoptotic Bim, $\mathrm{BH} 3$ and $\mathrm{Bmf}$ during the UV induced apoptosis to release release them from sequestering myosinV motor and dynein complexes -only proteins Bim and Bmf to release them from sequestering dynein and myosin $\mathrm{V}$ motor complexes respectively [48]. Further more release of Bim and Bmf can activate Bax or Bax to intitate the apoptosis. [49].To neurtrilize the anti apoptotic activites of BCL2 and BCL2 homologs, the Jnk phosphorylated Bim can bind and neutralize the anti apoptoic-activites of BCL2 homologs and BCL2[50].

JNK can also promote apoptosis through the phosphorylation of pro- apoptotic protein Bad JNK has also been shown to promote apoptosis through the phosphorylation of the pro-apoptotic protein Bad [51].It was reported that JNK phosphorylates the Ser 123 of BAD and promotes its apoptotic effect of BAD in primary granule neurons of rat cerebellum [51]. During the absence of apoptotic stimuli, the pro survical kineases PAK-1 AK1 and PKA inhibit apoptotic activity of BAD by phosphorylating it at Ser112/136/155, following which the phosphorylaed BAD is sequestered by 14-3-3 family of proteints. Through the phosphorylation of the BAD at Ser 128,an event which inhibits Bad 14-3-3 interaction JNK promotes apoptosis moreover it also potentiates the mechanism through phosphorylating 14-3-3 protein at Ser184 following which 14-3-3 relaease the sequestered Bad [52]. Some evidance showes that JNK promotes apoptosis through the direct phosphorylation of the Bcl-2 to inhibit its anti apoptotic activity.During the paclitaxel induced apoptosis of the breast cancer cells, it has been shown that JNK is activated and the activated JNK phosphorylates Bcl2 at Ser70 to induce apoptosis[53].

\section{JNK in Extrinsic Apoptotic Pathway}

Extrinsic apoptotic pathway can be induced through the apoptotic signaling initiated through the activation of death receptors, such as Trail-R1 (DR4), CD95 (Fas,APO-1), TRAIL-R2 (DR5), TNFR1, DR3 (TRAMP) and DR6 through their respective ligands [54].Those death receptors which are activated recruit the adaptor molecules such as FAS assoicated death domain protein that in turn activates caspase 8, which directly activates the caspase 3 and caspase 7 that initiates apoptosis. Activated death receptors recruit the adaptor molecules such as FAS associated death domain protein, which activates caspase 8 . Activated caspase 8 directly activates caspase 3 and caspase 7 that initiates apoptosis.However, some of the death receptors can also induce activation of JNKs. Throgh the AP1 mediated increased expression of death receptors, pro- apoptotic proteins such as Bim, Bak[55], Bax [56], Trail-R2 [57] and ligands the JNKs contribute to extrinsic pathway. In HeLa cells TNF- $\alpha$ induced apoptosis, JNK mediated phosphorylation causes the cleavage of Bid.The $21 \mathrm{kDa}$ cleaved fragment of the Bid translocates to mitochondria and selectively induces the release of OMI, SMAC/DIABLO, the two of the mitochondria intermembrane proteins that can inhibit the apoptotic protein XIAP, an inhibitor of caspase 8. In addition, OMI inhibits cellular-inhibitor of apoptosis protein 1 or cIAP1, another inhibitor of caspase 8 [58]. Inhibition of XIAP and cIAP1 leads to the activation of the executioner caspases 3 and 7, leading to apoptosis [59]. Even in the absence of such a cleavage, JNK-phosphorylated full length Bid translocates to mitochondria and potentiates extrinsic signaling during TNFinduced apoptosis in PC12 cells [60].Cumulatively, the multiple signaling inputs generated by JNK promotes extrinsic apoptotic pathway

\section{JNK signaling in heart failure}

Heterotrimeric guanine nucleotide-binding proteins (G proteins) of the $\mathrm{Gq}$ family transduce signals from a different of widely expressed membrane receptors to generate diverse, tissue-specific effects [61]. In variety of target tissues, receptor mediated activation of Gq regulates physiological responses such as contraction and secretion. A role for Gq-coupled receptors in regulation of cell growth has become apparent only more recently[62]. Cardiac muscle expresses Gq-coupled receptors that do not appear to play a primary role in modulating cardiac contractile function. Rather, the relevant physiological role for Gq-coupled receptor agonists may be stimulation of cardiac hypertrophy. Indeed, multiple Gqcoupled receptor agonists stimulate hypertrophy of cultured neonatal rat cardiac myocytes [63]. In the intact heart, cardiac hypertrophy is typically a compensatory response to increased hemodynamic load. The resulting increase in cardiac mass improves cardiac performance in the short term by reducing wall stress [64]. When the hemodynamic load is not relieved however, the hypertrophied heart ultimately dilates and fails in a phenomenon termed "decompensation." A role for Gq-signaling in the development and decompensation of hypertrophy is supported by theeffects of transgenic overexpression of $\alpha 1$ adrenergic and angiotensin II receptors in cardiomyocytes [65]. In fact, heterozygous transgenic overexpression of Gaq in cardiomyocytes induces cardiac 
enlargement with many of the molecular, structural and functional characteristics of pressure overload hypertrophy [66].higher level of Gaq overexpression in dual heterozygous mice causes a lethal dilated congestive cardiomyopathy with myocyte loss in the absence of inflammation, suggesting a role for apoptosis. Although cardiomyocyte apoptosis has been implicated in human cardiomyopathy [67] and animal models of myocardial injury [68], a role for Gq signaling in apoptotic cardiomyocyte death has not been explored, and the relationship between apoptosis and cardiac failure has not been defined adequately. It was explore the role of Gq signaling in cardiomyocyte hypertrophy and apoptosis by using cultured adenovirus-infected neonatal cardiac myocytes or transgenic mice with enhanced $\mathrm{G}$ a q signaling. The results demonstrate that sustained high level activation of Gaq can produce apoptotic cardiomyocyte death and can lead to heart failure.In the heart, JNK is activated in response to a variety of pathological insults such as hypertension, ischemia/reperfusion injury, and myocardial infarction [69]. Several studies have implicated JNK signaling as a key mediator of pathological cardiac remodeling and heartfailure.An important role for JNK signaling has been demonstrated in cardiac hypertrophy [70] as well as cardiomyocyte apoptosis[71]. JNK activation to a specific aspect of pathological cardiac remodeling, stress-induced down-regulation of the gap junction protein connexin 43 (Cx43) [72]. Loss of gap junctions is a common feature of the failing heart [73].

\section{JNK signaling and apoptosis in the heart}

JNK activation begin at the cell membrane by the activation of the Cdc42/Ras/Rac.these these GTP binding proteins directly induce MAPK kinase kinase 1,2,3 and apoptosis signal- regulating kinase-1(ASK1) activation which in turn promote phosphorylation and activation of MAPK kinases, MKK4 and MKK7 that directly phosphorylate and activate JNK[77].There are three different types JNK genes that encode over $10 \mathrm{JNK}$ isoforms through alternative splicing[78]. JNK 1 and JNK2 can be produced in mouse tissues including the heart, while JNK3 can not be produced in heart[79].JNKs can be activated by the vareity of stree and mitogenic stimuli, such as growth factors, cytokines, UV irradiation, toxic compounds, osmotic shock, hypoxia, ischemia and oncogenes[80].Activation of JNK results in activation of several trascription factors such as the as c-jun activating trascription factor-2 (ATF2),P53,Elk1, nuclear factor of activated T-cells(NFAT) and Sap-1[77].JNK is the first MAPK activated in the rat heart by production of chronic angiotensin II infusion and in culture by mechanial stretch[81].It was shown that JNK can be activated by Ras in transgenic hearts, by ischemia reperfusion injury and by pressure overload[82]. Moreover JNK can aslo activated by the Gaq couple receptor agonist, such as phenylephrine(PE) which is required for atrial natriuretic factor(ANF) promoter activity in cardiac myocytes[82]. Activation of JNK signaling pathway by expression of an activated MKK7 mutant protein in cardiac myocytes induced the characteristic feature of hypertrophy in vitro[83]. Furthermore, expression of MKK4 which is dominant negative attenuated the hypertrophic response of neonatal rat in culture of endothelin 1 in cardiomyocytes[84].Similarly the adenoviral gene transformation of MKK4 protein to the adult rat heart blocked activation of JNK and attenuate the cardiac hypertrophy by pressure overload[85].However, some recent studies in mekk1 gene targeted mice or jnk1 or jnk2 gene targeted mice showed no positive correlation with the cardiac hypertrophic respose in vivo[86].

Although loss of MEKK1 attenuated hypertrophy, cardiomyopathy associaged with Gaq overexpression[87],although loss of mekk1 reduced hypertrophic cardiomyopathy associated with Gaq overexpression [87]. While some disagreement are exists to the hypertrophic regulatory role for JNK signaling in the heart. In some studies it has shown a critical role for JNK factors and their upstream activators as affectors of apoptosis in mamalian cells. For example, the nul fibroblasts JNK were demonstrated to be deficient in mitochondria driven apoptosis, suggest that jnk has critical role in this process[88].Similarly, jnk3 null mice showed significant reductions in excitoxicity-induced hippocampal neuron apoptosis [89]. Jnk 2 null mice showed the defect in apoptosis in immature thymocytes[90]. While jnk1 or jnk 2 double null mice indicated alterations in region specific apoptosis in developing mouse brain [91]. Up stream of jnk1/2/3, ask null cells indicated significant impairment in $\mathrm{H} 2 \mathrm{O} 2$ and TNF a induced cell death[92].Null embryonic mekk1 stem cells can be differentiated in to the cardiac myocytes indicated enhanced oxidative stress induced apoptosis[93].Moreover, jnk2 null mice also showed apoptotic defects in immature thymocytes [90], Consistent with the observation that mekk1 null mice have more cardiac apoptosis following pressure overload stimulation[71]. However,the results observed in mekk1 deficient cells suggest that the JNK signaling branch can serve a protective role, in contrast to data obtained in ask1, mekk1, andjnkgene-targeted mice.However,MEKK1can also influence ERK activation, as well as effect other regulatory events that are independent of JNK signaling,suggesting that greater emphasis should be placed on the jnk gene targeting studies for cause-and-effect assessment [94]. Further investigation into the causality between JNK signaling and cardiac myocyte apoptosis has revealed additional discordance. For example, expression of dominant negative JNK1 or dominant-negative MKK4 in cultured cardiacmyocytes increased nitricoxide- 
inducedcardiomyocyte apoptosis, suggesting a protective role for JNK signaling [95]. Consistent with this study, ischemia-reperfusion induced apoptosis in cultured neonatal cardiomyocytes was increased by expression of JNK inhibitory mutants [96]. In contrast, JNK1 inhibition was recently reported to actually protect cardiac myocytes from ischemia-induced apoptosis, while JNK2 inhibition had no effect [96]. Similarly, inhibition of JNK in H9c2 myocytes blocked apoptosis induced by stressst imulation [97], while infection of adult cardiacmyocytes with a dominant-negative JNK-expressing adenovirus antagonized H2O2-induced apoptosis[98].Upstream of JNK signaling,targeted deletion of ask1 in mice attinuated the myocyte apoptosis following pressure overload and myocardial infarction, however the expression of an activated ASK1 mutant protein stimulated myocyte apoptosis in culture[99]. The b-adrenergic stimulated apoptosis of cultured adult cardiomyocyte was inhibited through expression of dominant negative JNK by a mitochondrial dependent mechanism[100].In a study by, Aoki et al. It showed that activated JNK and MKK4 localized to the mitochondria,induced cytochrome c release, and promoted apoptosis of cardiac myocytes [101]. Baines et al. Reported that fraction of JNK protein was localized to mitochondria [102], and is consistent with an earlier observation made in fibroblasts, whereas JNK was required for cytochrome c release and mitochondrial-driven cell death [43]. Apoptosis can be induced by JNK signaling through a transcription dependent mechanism which involves the activation of the c-jun and induction of pro apoptotic genes[103].It was also reported that JNK directly phoshphorylate the pro- survival factor Bcl-2, restuling in its activation[104]. With respect to the Bcl-1 family JNK can directly phosphorylate the serine 128 on the pro apoptotic factor Bad, enhancing cell the death through the intrinsic pathway [105]. JNK activation was also recently shown to directly activate Bid cleavage, independent of caspase-8, thus further facilitating the mitochondrial death pathway [106].It was also reported that JNK directly phsophorylate two additional pro -apoptotic memebers of the BH3- only subgroup of bim bcl-2 and bmf, facilitating their translocation to mitchondria[107].

\section{Conclusion:-}

We found out that the JNK signaling pathway activated by the biologic stimuli such as the TNF, Gowth factors ,Stress ,Cytokines, ischemia and hypoxia the overexpression of the following molecule is belived that it causes to the progression of HF by virtue of the deleterious effects they exert on the heart and the circulation. JNK is a master protein kinase that plays an important role in osteoblast proliferation, differentiation and apoptosis. An overview of apoptotic pathway indicates that JNK signaling is involved in the extrinsic apoptotic pathway initiated by death receptors as well as the intrinsic pathway initiated at the mitochondria. In response to both the extrinsic and intrinsic apoptotic stimuli, JNK plays an essential role through its ability to interact and modulate the activities of diverse pro- and anti-apoptotic proteins.

\section{References:-}

1. Widmann, C., Gibson, S., Jarpe, M.B., and Johnson, G.L. 1999. Mitogenactivated protein kinase: conservation of a three-kinase module from yeast to human. Physiol. Rev.79:143-180.

2. Sugden, P.H., and Clerk, A. 1998. "Stress-responsive" mitogen-activated protein kinases (c-Jun N-terminal kinases and p38 mitogen-activated protein kinases) in the myocardium. Circ. Res.83:345-352.

3. Davis RJ. Signal transduction by the JNK group of MAP kinases. Cell 2000; 103:239-52; PMID:11057897; http://dx.doi.org/10.1016/ S0092-8674(00)00116-1

4. Weston CR, Davis RJ. The JNK signal transduction pathway. Curr Opin Genet Dev 2002; 12:1421; PMID:11790549; http://dx.doi.org/10.1016/ S0959-437X(01)00258-1

5. Lin A, Dibling B. The true face of JNK activation in apoptosis. Aging Cell. 2002; 1:112-116. [PubMed: 12882340]

6. Liu J, Lin A. Role of JNK activation in apoptosis: a double-edged sword. Cell Res. 2005; 15:36-42. [PubMed: 15686625]

7. Devary Y, Gottlieb RA, Lau LF, Karin M. Rapid and preferential activation of the c-jun gene during the mammalian UV response. Mol Cell Biol 1991; 11:2804-11; PMID:1901948

8. Hibi M, Lin A, Smeal T, Minden A, Karin M. Identification of an oncoprotein- and UV-responsive protein kinase that binds and potentiates the c-Jun activation domain. Genes Dev. 1993; 7:2135- 2148. [PubMed: 8224842]

9. Fanger GR, Gerwins P, Widmann C, Jarpe MB, Johnson GL. MEKKs, GCKs, MLKs, PAKs, TAKs, and tpls: upstream regulators of the c-Jun aminoterminal kinases? Curr Opin Genet Dev 1997; 7:6774; PMID:9024636; http://dx.doi.org/10.1016/ S0959-437X(97)80111-6

10. Yu C, Minemoto Y, Zhang J, Liu J, Tang F, Bui TN, Xiang J, Lin A. JNK suppresses apoptosis via phosphorylation of the proapoptotic Bcl-2 family protein BAD. Mol Cell 2004; 13:329-40; PMID:14967141; 
11. Leppä S, Bohmann D. Diverse functions of JNK signaling and c-Jun in stress response and apoptosis. Oncogene 1999; 18:6158-62; PMID:10557107; http://dx.doi.org/10.1038/sj.onc.1203173

12. Yu C, Minemoto Y, Zhang J, Liu J, Tang F, Bui TN, Xiang J, Lin A. JNK suppresses apoptosis via phosphorylation of the proapoptotic Bcl-2 family protein BAD. Mol Cell 2004; 13:329-40; PMID:14967141; http://dx.doi.org/10.1016/S1097-2765(04)00028-0

13. Westwick JK, Weitzel C, Minden A, Karin M, Brenner DA. Tumor necrosis factor alpha stimulates AP-1 activity through prolonged activation of the c-Jun kinase. J Biol Chem. 1994; 269:26396- 26401. [PubMed: 7929360]

14. Cano E, Hazzalin CA, Mahadevan LC. Anisomycin-activated protein kinases p45 and p55 but not mitogenactivated protein kinases ERK-1 and -2 are implicated in the induction of c-fos and c-jun. Mol Cell Biol. 1994; 14:7352-7362. [PubMed: 7935449]

15. Elmore S. Apoptosis: a review of programmed cell death. Toxicol Pathol. 2007; 35:495-516. [PubMed: 17562483]

16. Davis RJ. Signal transduction by the JNK group of MAP kinases. Cell. 2000; 103:239-252. [PubMed: 11057897]

17. Johnson GL, Nakamura K. The c-jun kinase/stress-activated pathway: regulation, function and role in human disease. Biochim Biophys Acta. 2007; 1773:1341-1348. [PubMed: 17306896]

18. Dhanasekaran DN, Kashef K, Lee CM, Xu H, Reddy EP. Scaffold proteins of MAP-kinase modules. Oncogene. 2007; 26:3185-3202. Review. [PubMed: 17496915]

19. Dhanasekaran N, Reddy EP. Signaling by dual specificity kinases. Oncogene. 1998; 17:1447-1755. [PubMed: 9779990

20. Kyriakis JM, Brautigan DL, Ingebritsen TS, Avruch J. Pp54 microtubule-associated protein-2 kinase requires both tyrosine and serine/threonine phosphorylation for activity. J Biol Chem 1991;266:10043-6.

21. Bode,A.M.,andDong,Z.(2007).ThefunctionalcontrarietyofJNK.MolCarcinog. $\quad 46, \quad 591-598 . \quad$ doi: 10.1002/mc. 20348

22. Avruch, J. (2007). MAP kinase pathways: the first twenty years. Biochim. Biophys. Acta 1773, 1150-1160. doi: 10.1016/j.bbamcr.2006.11.006

23. Patterson, K. I., Brummer, T., O’Brien, P. M., and Daly, R. J. (2009). Dualspecificity phosphatases: critical regulators with diverse cellular targets. Biochem. J. 418, 475-489. doi: 10.1042/BJ20082234

24. Wagner, E. F., and Nebreda, A. R. (2009). Signal integration by JNK and p38 MAPK pathways in cancer development. Nat. Rev. Cancer 9, 537-549. doi: 10.1038/nrc2694

25. Fanger GR, Gerwins P, Widmann C, Jarpe MB, Johnson GL. MEKKs, GCKs, MLKs, PAKs, TAKs, and tpls: upstream regulators of the c-Jun amino-terminal kinases? Curr Opin Genet Dev. 1997; 7:67-74. [PubMed: 9024636]

26. Wagner, E. F., and Nebreda, A. R. (2009). Signal integration by JNK and p38 MAPK pathways in cancer development. Nat. Rev. Cancer 9, 537-549. doi: 10.1038/nrc2694

27. Gupta,S.,Barrett,T.,Whitmarsh,A.J.,Cavanagh,J.,Sluss,H.K.,Dérijard,B.,etal. (1996). Selective interaction of JNK protein kinase isoforms with transcription factors. EMBO J. 15, 2760-2770.

28. Davis, R. J. (2000). Signal transduction by the JNK group of MAP kinases. Cell 103, 239-252. doi: 10.1016/S0092-8674(00)00116-1

29. Bogoyevitch MA, Gillespie-Brown J, Ketterman AJ, Fuller SJ, Ben-Levy R,Ashworth A, et al. Stimulation of the stress-activated mitogen-activated proteinkinase subfamilies in perfused heart. p38/RK mitogen-activated protein kinases and c-Jun N-terminal kinases are activated by ischemia/reperfusion. Circ Res 1996;79:162-73.

30. Goldsmith ZG, Dhanasekaran DN. G protein regulation of MAPK networks. Oncogene 2007;26:3122-42.

31. Rose BA, Force T, Wang Y. Mitogen-activated protein kinase signaling in theheart: angels versus demons in a heart-breaking tale. Physiol Rev 2010;90:1507-4

32. Pandur P, Lasche M, Eisenberg LM, Kuhl M. Wnt-11 activation of a non-canonical Wnt signalling pathway is required for cardiogenesis. Nature 2002;418:636-41

33. Sugden PH, Clerk A. "Stress-responsive" mitogen-activated protein kinases(c-Jun N-terminal kinases and p38 mitogen-activated protein kinases) in the myocardium. Circ Res 1998;83:345-52.

34. Knight RJ, Buxton DB. Stimulation of c-Jun kinase and mitogen-activated protein kinase by ischemia and reperfusion in the perfused rat heart. Biochem Biophys Res Commun 1996;218:83-8.

35. Pellieux, C., Sauthier, T., Aubert, J. F., Brunner, H. R., and Pedrazzini, T. (2000) J. Hypertens. 18, 1307-1317

36. Wang, Y., Su, B., Sah, V. P., Brown, J. H., Han, J., and Chien, K. R. (1998) J. Biol. Chem. 273, 5423-542

37. agents. Cancer Lett. 2014; 344:174-179. 37. Chen YR, Meyer CF and Tan TH. Persistent activation of c-Jun Nterminal kinase 1 (JNK1) in gamma radiationinduced apoptosis. J Biol Chem. 1996; 271:631-63 
38. Tournier C, Hess P, Yang DD, Xu J, Turner TK, Nimnual A, Bar-Sagi D, Jones SN, Flavell RA and Davis RJ. Requirement of JNK for stress-induced activation of the cytochrome c-mediated death pathway. Science. 2000; 288:870-874

39. Yang DD, Kuan CY, Whitmarsh AJ, Rincon M, Zheng TS, Davis RJ, Rakic P and Flavell RA. Absence of excitotoxicity-induced apoptosis in the hippocampus of mice lacking the Jnk3 gene. Nature. 1997; 389:865-870.

40. Chang L and Karin M. Mammalian MAP kinase signalling cascades. Nature. 2001; 410:37-40.

41. Turjanski AG, Vaque JP and Gutkind JS. MAP kinases and the control of nuclear events. Oncogene. 2007; 26:32403253.

42. Fan M and Chambers TC. Role of mitogen-activated protein kinases in the response of tumor cells to chemotherapy. Drug Resist Updat. 2001; 4:253-267.

43. Johnson GL and Nakamura K. The c-jun kinase/stressactivated pathway: regulation, function and role in human disease. Biochim Biophys Acta. 2007; 1773:1341-1348.

44. Bjorkblom B, Vainio JC, Hongisto V, Herdegen T, Courtney MJ and Coffey ET. All JNKs can kill, but nuclear localization is critical for neuronal death. J Biol Chem. 2008; 283:19704-19713

45. Fuchs SY, Adler V, Buschmann T, Yin Z, Wu X, Jones SN and Ronai Z. JNK targets p53 ubiquitination and degradation in nonstressed cells. Genes Dev. 1998; 12:2658-2663

46. Oleinik NV, Krupenko NI and Krupenko SA. Cooperation between JNK1 and JNK2 in activation of p53 apoptotic pathway. Oncogene. 2007; 26:7222-7230.

47. Jones EV, Dickman MJ and Whitmarsh AJ. Regulation of p73-mediated apoptosis by c-Jun N-terminal kinase. Biochem J. 2007; 405:617-623

48. Lei K, Nimnual A, Zong WX, Kennedy NJ, Flavell RA, Thompson CB, Bar-Sagi D and Davis RJ. The Bax subfamily of Bcl2-related proteins is essential for apoptotic signal transduction by c-Jun NH(2)-terminal kinase. Mol Cell Biol. 2002; 22:4929-4942.

49. Mandal M, Olson DJ, Sharma T, Vadlamudi RK and Kumar R. Butyric acid induces apoptosis by up-regulating Bax expression via stimulation of the c-Jun N-terminal kinase/ activation protein-1 pathway in human colon cancer cells. Gastroenterology. 2001; 120:71-78.

50. Tsuruta F, Sunayama J, Mori Y, Hattori S, Shimizu S, Tsujimoto Y, Yoshioka K, Masuyama N and Gotoh Y. JNK promotes Bax translocation to mitochondria through phosphorylation of 14-3-3 proteins. EMBO J. 2004; 23:1889-1899.

51. Adams JM and Cory S. The Bcl-2 apoptotic switch in cancer development and therapy. Oncogene. 2007; 26:13241337.

52. Lei K and Davis RJ. JNK phosphorylation of Bim-related members of the Bcl2 family induces Bax-dependent apoptosis. Proc Natl Acad Sci U S A. 2003; 100:2432-2437.

53. Marani M, Tenev T, Hancock D, Downward J and Lemoine NR. Identification of novel isoforms of the BH3 domain protein Bim which directly activate Bax to trigger apoptosis. Mol Cell Biol. 2002; 22:3577-3589.

54. Hubner A, Barrett T, Flavell RA and Davis RJ. Multisite phosphorylation regulates Bim stability and apoptotic activity. Mol Cell. 2008; 30:415-425.

55. Donovan N, Becker EB, Konishi Y and Bonni A. JNK phosphorylation and activation of BAD couples the stressactivated signaling pathway to the cell death machinery. J Biol Chem. 2002; 277:40944-40949

56. Sunayama J, Tsuruta F, Masuyama N and Gotoh Y. JNK antagonizes Akt-mediated survival signals by phosphorylating 14-3-3. J Cell Biol. 2005; 170:295-304.

57. Yamamoto K, Ichijo H and Korsmeyer SJ. BCL-2 is phosphorylated and inactivated by an ASK1/Jun Nterminal protein kinase pathway normally activated at G(2)/M. Mol Cell Biol. 1999; 19:8469-8478.

58. Walczak H. Death receptor-ligand systems in cancer, cell death, and inflammation. Cold Spring Harb Perspect Biol. 2013; 5:a008698.

59. Jin HO, Park IC, An S, Lee HC, Woo SH, Hong YJ, Lee SJ, Park MJ, Yoo DH, Rhee CH and Hong SI. Upregulation of Bak and Bim via JNK downstream pathway in the response to nitric oxide in human glioblastoma cells. J Cell Physiol. 2006; 206:477-486.

60. Chen YJ, Liu WH, Kao PH, Wang JJ and Chang LS. Involvement of p38 MAPK- and JNK-modulated expression of Bcl-2 and Bax in Naja nigricollis CMS-9induced apoptosis of human leukemia K562 cells. Toxicon. 2010; 55:1306-1316

61. Zou W, Liu X, Yue P, Zhou Z, Sporn MB, Lotan R, Khuri FR and Sun SY. c-Jun NH2-terminal kinasemediated upregulation of death receptor 5 contributes to induction of apoptosis by the novel synthetic triterpenoid methyl-2cyano-3,12-dioxooleana-1, 9-dien-28-oate in human lung cancer cells. Cancer Res. 2004; 64:7570-7578. 
62. Silke $\mathbf{J}$ and Meier P. Inhibitor of apoptosis (IAP) proteinsmodulators of cell death and inflammation. Cold Spring Harb Perspect Biol. 2013; 5.

63. Deng Y, Ren X, Yang L, Lin Y and Wu X. A JNK-dependent pathway is required for TNFalpha-induced apoptosis. Cell. 2003; 115:61-70

64. Prakasam A, Ghose S, Oleinik NV, Bethard JR, Peterson YK, Krupenko NI and Krupenko SA. JNK1/2 regulate Bid by direct phosphorylation at Thr59 in response to ALDH1L1. Cell Death Dis. 2014; 5:e1358

65. Simon, M. I., Strathmann, M. P. \& Gautam, N. (1991) Science 252, 802-808.

66. Post, G. R. \& Brown, J. H. (1996) FASEB J. 10, 741-749.

67. Sadoshima, J.-I. \& Izumo, S. (1993) Circ. Res. 73, 413-423.

68. 7. Grossman,W.,Jones,D.\&McLaurin,L.P.(1975)J.Clin.Invest. 55, 56-64

69. Milano, C. A., Dolbert, P. C., Rockman, H. A., Bond, R. A., Venable,M.E.,Allen,L.F.\&Lefkowitz,R.J.(1994)Proc.Natl. Acad. Sci. USA 91, 10109-10113

70. D’Angelo, D. D., Sakata, Y., Lorenz, J. N., Boivin, G. P., Walsh, R. A., Liggett, S. B. \& Dorn G. W., II (1997) Proc. Natl. Acad. Sci. USA 94, 8121-8126

71. Narula, J., Haider, N., Virmani, R., DiSalvo, T. G., Kolodgie, F. D., Hajjar, R. J., Schmidt, U., Semigran, M. J., Dec, G. W. \& Khaw, B.-A. (1996) N. Engl. J. Med. 335, 1182-1189.

72. Fliss, H. \& Gattinger, D. (1996) Circ. Res. 79, 949-956.

73. Pellieux, C., Sauthier, T., Aubert, J. F., Brunner, H. R., and Pedrazzini, T. (2000) J. Hypertens. 18, 1307-1317

74. Wang, Y., Su, B., Sah, V. P., Brown, J. H., Han, J., and Chien, K. R. (1998) J. Biol. Chem. 273, 5423-5426

75. Aoki, H., Kang, P. M., Hampe, J., Yoshimura, K., Noma, T., Matsuzaki, M., and Izumo, S. (2002) J. Biol. Chem. 277, 10244-10250

76. Petrich, B. G., Gong, X., Lerner, D. L., Wang, X., Brown, J. H., Saffitz, J. E., and Wang, Y. (2002) Circ. Res. 91, 640-647

77. Barker, R. J., and Gourdie, R. G. (2002) Circ. Res. 91, 556-558

78. Molkentin JD, Dorn 2nd GW. Cytoplasmic signaling pathways that regulate cardiac hypertrophy.Annu Rev Physiol 2001;63:391-426

79. GarringtonTP, Johnson GL. Organization and regulation of mitogenactivated protein kinase signaling pathways. Curr Opin Cell Biol 1999;11:211-8.

80. Lenormand P, Sardet C, Pages G, L'Allemain G, BrunetA, Pouyssegur J. Growth factors induce nuclear translocation of MAP kinases (p42mapkandp44mapk)butnotoftheiractivatorMAPkinasekinase (p45mapkk) in fibroblasts. J Cell Biol 1993;122:1079-88.

81. Davis R. Signal transduction by the JNK group of MAP kinase. Cell 2000;103:239-52

82. GuptaS,BarrettT, WhitmarshAJ,CavanaghJ,SlussHK,DerijardB, et al. Selective interaction of JNK protein kinase isoforms with transcription factors. EMBO J 1996;15:2760-70.

83. Ito M, Yoshioka K, Akechi M, Yamashita S, Takamatsu N, Sugiyama K, et al. JSAP1, a novel Jun N-terminal protein kinase (JNK)bindingproteinthatfunctionsasascaffoldfactorintheJNKsignaling pathway. Mol Cell Biol 1999;19:7539-48

84. Johnson NL, Gardner AM, Diener KM, Lange-Carter CA, Gleavy J, Jarpe MB, et al. Signal transduction pathways regulated by mitogenactivated/extracellular response kinase kinase kinase induce cell death. J Biol Chem 1996;271:3229-37.

85. YanoM,KimS,IzumiY,YamanakaS,IwaoH.Differentialactivation of cardiac c-Jun amino-terminal kinase and extracellular signalregulated kinase in ang II-mediated hypertension. Circ Res 1998;83: 752-60.

86. RamirezMT,SahVP,ZhaoXL,HunterJJ,ChienKR,BrownJH.The MEKK-JNK pathway is stimulated by aadrenergic receptor and ras activationandisassociatedwithinvitroandinvivocardiachypertrophy. J Biol Chem 1997;72:14057-61.

87. WangY, Su B, SahVP, Brown JH, Han J, Chien KR. Cardiac hypertrophy induced by mitogen-activated protein kinase kinase 7, a specific activator for c-Jun NH2-terminal kinase in ventricular muscle cells. J Biol Chem 1998;273:5423-6.

88. Choukroun G, Hajjar R, Kyriakis JM, Bonventre JV, Rosenzweig A. Force T. Role of the stress-activated protein kinases in endothelininducedcardiomyocytehypertrophy.JClinInvest 1998;102:1311-20.

89. ChoukrounG,HajjarR,FryS,DelMonteF,HaqS,GuerreroJL,etal. Regulation of cardiac hypertrophy in vivo by the stress-activated proteinkinases/c-JunNH(2)-terminalkinases.JClinInvest1999;104: 391-8.

90. Sadoshima J, Montagne O, Wang Q,Yang G, Warden J, Liu J, et al. The MEKK1-JNK pathway plays a protective role in pressure overloadbutdoesnotmediatecardiachypertrophy.JClinInvest2002;110: 271-9.

91. MinaminoT,YujiriT,TeradaN,TaffetGE,MichaelLH,JohnsonGL, et al. MEKK1 is essential for cardiac hypertrophy and dysfunction induced by Gq. Proc NatlAcad Sci USA 2002;99:3866-71. 
92. Tournier C, Hess P, Yang DD, Xu J, Turner TK, Nimnual A, et al. Requirement of JNK for stress-induced activation of the cytochrome c-mediated death pathway. Science 2000;288:870-4.

93. Yang DD, Kuan CY,WhitmarshAJ, Rincon M, Zheng TS, Davis RJ, et al. Absence of excito toxicity-induced apoptosis in the hippocampus of mice lacking the Jnk3 gene. Nature 1997;389:865-70.

94. Sabapathy K, Hu Y, Kallunki T, Schreiber M, David JP, Jochum W, etal.JNK2isrequiredforefficientTcellactivationandapoptosisbut not for normal lymphocyte development. Curr Biol 1999;9:116-25.

95. Sabapathy K, Hu Y, Kallunki T, Schreiber M, David JP, Jochum W, etal.JNK2isrequiredforefficientTcellactivationandapoptosisbut not for normal lymphocyte development. Curr Biol 1999;9:116-25.

96. KuanCY,YangDD,SamantaRoyDR,DavisRJ,RakicP,FlavellRA. The Jnk1 and Jnk2 protein kinases are required for regional specific apoptosis during early brain development. Neuron 1999;22:667-76.

97. Minamino T, Yujiri T, Papst PJ, Chan ED, Johnson GL, Terada N. MEKK1 suppresses oxidative stress-induced apoptosis of embryonic stem cell-derived cardiac myocytes. Proc Natl Acad Sci USA 1999; 96:15127-32.

98. Yujiri T, Sather S, Fanger GR, Johnson GL. Role of MEKK1 in cell survivalandactivationofJNKandERKpathwaysdefinedbytargeted gene disruption. Science 1998;282:1911-4

99. Andreka P, Zang J, Dougherty C, Slepak TI, Webster KA, Bishopric NH. Cytoprotection by Jun kinase during nitric oxide-induced cardiac myocyte apoptosis. Circ Res 2001;88:305-12.

100.Dougherty CJ, Kubasiak LA, Prentice H, Andreka P, Bishopric NH, WebsterKA.Activationofc-JunNterminalkinasepromotessurvival of cardiac myocytes after oxidative stress. Biochem J 2002;362:561- 71 .

101.GabaiVL,MeriinAB,YaglomJA,WeiJY,MosserDD,ShermanMY. Suppression of stress kinase JNK is involved in HSP72-mediated protection of

102.Kwon SH, Pimentel DR, Remondino A, Sawyer DB, Colucci WS. H(2)O(2) regulates cardiac myocyte phenotype via concentrationdependent activation of distinct kinase pathways. J Mol Cell Cardiol 2003;35:61521.

103. Yamaguchi O, Higuchi Y, Hirotani S, Kashiwase K, Nakayama H, Hikoso S, et al. Targeted deletion of apoptosis signal-regulating kinase 1 attenuates left ventricular remodeling. Proc Natl Acad Sci USA 2003;100:15883-8.

104.Remondino A, Kwon SH, Communal C, Pimentel DR, Sawyer DB, Singh K, et al.b-adrenergic receptorstimulated apoptosis in cardiac myocytesismediatedbyreactiveoxygenspecies/c-JunNH2-terminal kinasedependent activation of the mitochondrial pathway. Circ Res 2003;92:136-8.

105.Aoki H, Kang PM, Hampe J,Yoshimura K, Noma T, Matsuzaki M, etal.Directactivationofmitochondrialapoptosismachinerybyc-Jun N-terminal kinase in adult cardiac myocytes. J Biol Chem 2002;277: 10244-50.

106.Baines CP, Zhang J, Wang GW, Zheng YT, Xiu JX, Cardwell EM, et al. Mitochondrial PKCe and MAPK form signaling modules in the murineheart:enhanced mitochondrial PKCe-MAPK interactionsand differentialMAPKactivationinPKCe-inducedcardioprotection.Circ Res 2002;90:390-7.

107.Xia Z, Dickens M, Raingeaud J, Davis RJ, Greenberg ME. Opposing effects of ERK and JNK-p38 MAP kinases on apoptosis. Science 1995;270:1326-31

108. Yamamoto K, Ichijo H, Korsmeyer SJ. Bcl-2 is phosphorylated and inactivated by an ASK1/Jun N-terminal protein kinase pathway normally activated at G(2)/M. Mol Cell Biol 1999;19:8469-78.

109.Donovan N, Becker EB, Konishi Y, Bonni A. JNK phosphorylation and activation of Bad couples the stressactivated signaling pathway to the cell death machinery. J Biol Chem 2002;277:40944-9.

110.DengY, Ren X, Yang L, LinY, Wu X. A JNK-dependent pathway is required for TNFa-induced apoptosis. Cell 2003;115:61-70

111.LeiK,DavisRJ.JNKphosphorylationofBim-relatedmembersofthe Bcl2 family induces Bax-dependent apoptosis. Proc Natl Acad Sci USA 2003;100:2432-7. 